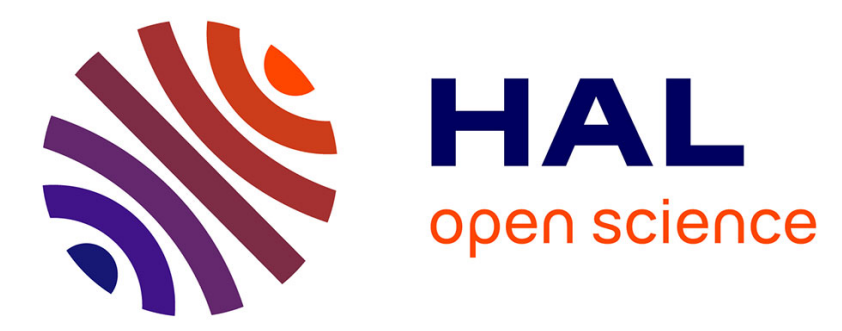

\title{
On the Asymptotic Performance of Receive Space Modulation in the Shadowing Broadcast Channel
}

Athanasios Stavridis, Marco Di Renzo, Peter Grant, Harald Haas

\section{To cite this version:}

Athanasios Stavridis, Marco Di Renzo, Peter Grant, Harald Haas. On the Asymptotic Performance of Receive Space Modulation in the Shadowing Broadcast Channel. IEEE Communications Letters, 2016, 20 (10), pp.2103 - 2106. 10.1109/LCOMM.2016.2592498 . hal-01880147

\section{HAL Id: hal-01880147 \\ https://hal.science/hal-01880147}

Submitted on 7 Jul 2020

HAL is a multi-disciplinary open access archive for the deposit and dissemination of scientific research documents, whether they are published or not. The documents may come from teaching and research institutions in France or abroad, or from public or private research centers.
L'archive ouverte pluridisciplinaire HAL, est destinée au dépôt et à la diffusion de documents scientifiques de niveau recherche, publiés ou non, émanant des établissements d'enseignement et de recherche français ou étrangers, des laboratoires publics ou privés. 


\title{
On the Asymptotic Performance of Receive Space Modulation in the Shadowing Broadcast Channel
}

\author{
Athanasios Stavridis, Marco Di Renzo, Senior Member, IEEE, Peter M. Grant Fellow, IEEE, and \\ Harald Haas, Member, IEEE
}

\begin{abstract}
In this paper, the diversity order and coding gain of Receive Space Modulation (RSM) in the Multiple-Input MultipleOutput (MIMO) broadcast channel are derived by taking into account both small and large scale fading. The considered linear precoding method is Zero Forcing (ZF). Note that the proposed framework is directly applicable to the conventional spatially multiplexed broadcast channel. Based on the derived mathematical framework, a theoretical criterion is provided which determines the superiority between the deployment of RSM and Spatial MultipleXing (SMX) in the shadowing MIMO broadcast channel. Finally, the provided theoretical results are validated via Monte Carlo simulation results.
\end{abstract}

Index Terms-MIMO, broadcast channel, spatial modulation, $\mathrm{ZF}$ precoding, large and small scale fading.

\section{INTRODUCTION}

Spatial Modulation (SM) is a multi-antenna communication technique which aims to minimize complexity and enhance Energy Efficiency (EE) [1-3]. Due to its potential, SM has been studied and incorporated in a wide range of communication scenarios [4-9]. Such an example is Receive Space Modulation (RSM) [10]. In RSM, instead of spatially modulating binary information on the transmit antennas, one or more symbol streams are spatially modulated to the indices of the receive antennas. This is undertaken by using linear precoding with Channel State Information at the Transmitter (CSIT) and the appropriate signal formation $[10,11]$.

In this paper, we provide the diversity order and coding gain of RSM in the Multiple-Input Multiple-Output (MIMO) broadcast channel when Zero Forcing (ZF) precoding is deployed and both small and large scale fading are explicitly taken into account. Compared to [12], in this paper, we consider and analytically illustrate the effect of random shadowing on the performance of the MIMO broadcast channel, for practical system setups. To the authors' best knowledge, such a study is not available in the published research. In particular, a Rayleigh distribution is assumed for small scale fading while for large scale fading both shadowing and pathloss are considered. Due to the mathematical intractability of the lognormal model of shadowing, a gamma distribution is used

Professor H. Haas acknowledges support from the EPSRC under Established Career Fellowship grant EP/K008757/1.

A. Stavridis, P. M. Grant, and H. Haas are with the Li-Fi Research and Development Centre, Institute for Digital Communications, School of Engineering, The University of Edinburgh, Edinburgh, UK. E-mail: \{a.stavridis, Peter.Grant, h.haas\}@ed.ac.uk.

M. Di Renzo is with the Laboratoire des Signaux et Systèmes, CNRS, CentraleSupélec, Univ Paris Sud, Université Paris-Saclay, 3 rue Joliot Curie, Plateau du Moulon, 91192, Gif-sur-Yvette, France. E-mail: marco.direnzo@1ss.supelec.fr. to model shadowing $[13,14]$. The combination of Rayleigh distributed small scale fading with gamma or lognormal distributed large scale fading provides two special cases of the general composite fading channel model [15]. In addition, using mathematical arguments, the performance of RSM is compared against the corresponding performance of Spatial MultipleXing (SMX) in the broadcast channel. It is shown that, in some scenarios, RSM performs better than SMX in terms of Bit Error Rate (BER).

\section{SySTEM MOdEL}

In this paper, the focus is on a system where a Base Station (BS) with $N_{t}$ antennas serves $N_{u}$ geographically distributed users. Each user is equipped with $N_{r}$ antennas. The baseband representation for such a system is expressed as, $\mathbf{y}=\boldsymbol{\Xi}^{\frac{1}{2}} \mathbf{H P D x}+\mathbf{w}$, in a matrix form. Here, the $N_{u} N_{r} \times 1$ collective received vector of all users is given as, $\mathbf{y}=\left[\mathbf{y}_{1}^{T}, \ldots, \mathbf{y}_{N_{u}}^{T}\right]^{T}$, where $\mathbf{y}_{i}, i=1, \ldots, N_{u}$, corresponds to the $N_{r} \times 1$ received signal vector of the $i$-th user. The $N_{r} N_{u} \times N_{t}$ wireless channel is expressed as $\overline{\mathbf{H}}=\mathbf{\Xi}^{\frac{1}{2}} \mathbf{H}$, where $\boldsymbol{\Xi}=\operatorname{diag}\left(\boldsymbol{\Xi}_{1}, \ldots, \boldsymbol{\Xi}_{N_{u}}\right)$ and $\mathbf{H}=\left[\mathbf{H}_{1}^{H}, \ldots, \mathbf{H}_{N_{u}}^{H}\right]^{H}$ represent the large and small scale fading, respectively. Here, $\operatorname{diag}(\cdot, \ldots, \cdot)$ is a block diagonal matrix. Also, $\boldsymbol{\Xi}_{i}$ and $\mathbf{H}_{i}$ denote the matrices of large and small scale fading of the $i$-th user, respectively.

The diagonal large scale fading matrix of the $i$-th user is given as, $\boldsymbol{\Xi}_{i}=\xi_{i} \mathbf{I}_{N_{r}, N_{r}}$, where $\xi_{i}=X_{i} / r_{i}^{\alpha}$ denotes the composite effect of pathloss and shadowing. Here, $r_{i}=\bar{r}_{i} / r_{0}$ denotes the normalized distance between the $i$-th user and the transmitting BS. Also, $\bar{r}_{i}$ and $r_{0}$ are the actual and the reference distance, respectively. The pathloss exponent is denoted by $\alpha$, with $2 \leq \alpha \leq 6$. In this paper, the effect of random shadowing is modeled by a Random Variable (RV) $X_{i}$ which follows a gamma distribution. Therefore, the Probability Density Function (PDF) of $X_{i} \sim \operatorname{Gamma}\left(k_{i}, \Omega_{i}\right)$ is given as:

$$
f_{X_{i}}(x)=\frac{x^{k_{i}-1}}{\Gamma\left(k_{i}\right) \Omega_{i}^{k_{i}}} e^{-\frac{x}{\Omega_{i}}} H_{0}(x), \quad k_{i}, \Omega_{i}>0
$$

where, $k_{i}$ and $\Omega_{i}=\mathrm{E}_{X_{i}}\left[X_{i}\right] / k_{i}$ are the shape and scale parameters of the gamma distribution. Also, the variance of shadowing represented by $X_{i}$ is given as $\sigma_{X_{i}}^{2}=k_{i} \Omega_{i}^{2}$. Furthermore, $H_{0}(x)$ is the Heaviside step function defined as, $H_{0}(x)=0$ for $x<0$ and $H_{0}(x)=1$ for $x \geq 0$. Note that this type of modeling of shadowing has been extensively used in the published research in order to simplify the mathematical difficulty imposed by the log-normal shadowing model [14, 
16]. Furthermore, the small scale fading matrices $\mathbf{H}_{i}$ distribute as, $\mathbf{H}_{i} \sim \mathcal{C N}(\mathbf{0}, \mathbf{I})$.

Provided that perfect CSIT is available, the ZF precoder is given as, $\breve{\mathbf{P}}=\mathbf{H}^{H}\left(\mathbf{H H}^{H}\right)^{-1} \boldsymbol{\Xi}^{-\frac{1}{2}}$. In this case, the $N_{u} N_{r} \times N_{u} N_{r}$ block diagonal normalization matrix $\mathbf{D}$ can be expressed as, $\mathbf{D}=\boldsymbol{\Xi}^{\frac{1}{2}} \mathbf{d i a g}\left(\mathbf{D}_{1}, \ldots, \mathbf{D}_{N_{u}}\right)$, where $\mathbf{D}_{i}$ are diagonal matrices. In [12], it is shown that the $j$-th element, $j=1, \ldots, N_{r}$, of $\mathbf{D}_{i}$ is expressed as:

$$
d_{j}=\sqrt{\frac{1}{\left[\left(\mathbf{H H}^{H}\right)^{-1}\right]_{i j, i j}}} .
$$

The $N_{u} N_{t} \times 1$ collective signal vector $\mathbf{x}$ is expressed as $\mathbf{x}=\left[\mathbf{x}_{1}^{T}, \ldots, \mathbf{x}_{N_{u}}^{T}\right]^{T}$. Here, $\mathbf{x}_{i} \in \mathcal{B}_{i}, i=1, \ldots, N_{u}$, denotes the $N_{r} \times 1$ information carrying signal vector for the $i$-th user. The deployed transmission alphabet for the $i$-th user is denoted as $\mathcal{B}_{i}$. The $N_{u} N_{r} \times 1$ vector $\mathbf{w}=\left[\mathbf{w}_{1}^{T}, \ldots, \mathbf{w}_{N_{u}}^{T}\right]^{T} \sim \mathcal{C N}\left(\mathbf{0}, \sigma_{\mathbf{w}}^{2} \mathbf{I}\right)$ is the collective representation of the white Gaussian noise experienced by all users. Finally, $\mathbf{w}_{i}$ is the white Gaussian noise of the $i$-th user.

By incorporating the ZF precoder into the system baseband equation and focusing on the received signal of the $i$-th user, the following expression is obtained:

$$
\mathbf{y}_{i}=\boldsymbol{\Xi}_{i}^{\frac{1}{2}} \mathbf{D}_{i} \mathbf{x}_{i}+\mathbf{w}_{i} .
$$

The observation of (3) shows that the selection of the elements of the alphabet $\mathcal{B}_{i}, \mathbf{x}_{i}$, determines the way that binary information is conveyed to each user. In RSM, $1 \leq N_{s}<N_{r}$ parallel symbol steams are spatially modulated at the receive antennas of each user. In more detail, the alphabet of RSM has the following form, $\mathbf{x}_{i}=\left[\mathbf{0}_{1 l_{1}}, s_{1}, \mathbf{0}_{1 l_{i}}, \ldots, \mathbf{0}_{1 l_{i}}, s_{N_{s}}, \mathbf{0}_{1 l_{N_{s}-1}}\right]^{T}$. In RSM, due to the structure of $\mathbf{x}_{i}$, every symbol period, $k_{1}=N_{s} \log _{2}(M)$ bits are conveyed by $N_{s}$ symbols drawn from a conventional constellation $\mathcal{M}$. The order of $\mathcal{M}$ is denoted as $M$. In addition, given that $N_{s}<N_{r}$, the $N_{s}$ symbols can be placed into $\left(\begin{array}{l}N_{r} \\ N_{s}\end{array}\right)$ possible combinations in $\mathbf{x}_{i}$. Here, (.) denotes the binomial coefficient. Therefore, $k_{2}=\left\lfloor\log _{2}\left(\left(\begin{array}{c}N_{r} \\ N_{s}\end{array}\right)\right)\right\rfloor$ bits can be encoded on the positions of $s_{l}$, $l=1, \ldots, N_{s}$, in $\mathbf{x}_{i}$. In fact, (3) shows that the received signal of the $i$-th user is a scaled version of $\mathbf{x}_{i}$ degraded by noise. Hence, the positions of the non zero elements of $\mathbf{x}_{i}$ correspond to the antennas which receive a non zero signal, while, the rest of the antennas experience only noise. The spectral efficiency of RSM is $k_{\mathrm{RSM}}=k_{1}+k_{2}$ bits per channel use (bpcu).

SMX transmission can be considered as a special case of RSM. In SMX, it holds that $N_{s}=N_{r}$ and binary information is solely conveyed by the $N_{r}$ symbols drawn from $\mathcal{M}$. Thus, the spectral efficiency of SMX is $k_{\mathrm{SMX}}=N_{r} \log _{2}(M)$.

In this paper, the transmitted bits to the $i$-th user are recovered by deploying the following Maximum Likelihood (ML) detector, $\left(\tilde{\mathbf{x}}_{i}\right)=\arg \min _{\mathbf{x}_{i}}\left\|\mathbf{y}_{i}-\sqrt{\xi_{i}} \mathbf{D}_{i} \mathbf{x}_{i}\right\|_{2}^{2}$. Here, $\tilde{\mathbf{x}}_{i}$ is the detected symbol vector at the $i$-th user.

\section{ANALysis OF Diversity ORder AND CODING GAIN}

The derivation of the diversity order and coding gain of the $i$-th user can be obtained by expressing the high Signalto-Noise Ratio (SNR) approximation of its Symbol Error Rate
(SER) in the appropriate form [17]. Based on the union bound method, a high SNR approximation of the SER of the $i$-th user is given as [18]:

$$
\mathrm{SER}_{i}^{+\infty} \lesssim \frac{1}{\left|\mathcal{B}_{i}\right|} \sum_{\substack{\mathbf{x}_{i} \\ \hat{\mathbf{x}}_{i} \neq \mathbf{x}_{i}}} \sum_{\substack{\hat{\mathbf{x}}_{i} \\ \mathbf{x}_{i}}} P_{i}^{+\infty}\left(\mathbf{x}_{i} \rightarrow \hat{\mathbf{x}}_{i}, \gamma\right) .
$$

Here, $P_{i}^{+\infty}\left(\mathbf{x}_{i} \rightarrow \hat{\mathbf{x}}_{i}, \gamma\right)$ is the high SNR approximation of the Pairwise Error Probability (PEP) of transmitting the symbol vector $\mathbf{x}_{i}$ to the $i$-th user, while, its detector erroneously decides in favor of the symbol vector $\hat{\mathbf{x}}_{i}$. Also, $\gamma=1 / \sigma_{\mathbf{w}}^{2}$ is the transmit SNR. Here, it is assumed that $\mathrm{E}_{\mathbf{x}}\left[\|\mathbf{x}\|_{2}^{2}\right]=1$.

As shown in [12, Section V], the high SNR approximation of the PEP of the $i$-th user conditioned on the RV $X_{i}$, which represents the effect of large scale fading, is expressed as:

$$
P_{i}^{+\infty}\left(\mathbf{x}_{i} \rightarrow \hat{\mathbf{x}}_{i}, \gamma \mid X_{i}\right) \lesssim\left[\vartheta_{i} X_{i} \gamma\right]^{-N_{i} L}+o\left(\gamma^{-N_{i} L}\right) .
$$

In (5), it holds that $\vartheta_{i}=\lambda\left(\mathbf{x}_{i}, \hat{\mathbf{x}}_{i}\right) \sqrt[N_{i} L]{2} / 4 r_{i}^{a}$, where, $L=$ $N_{t}-N_{u} N_{r}+1$ and $N_{i}$ is the number of different elements between $\mathbf{x}_{i}$ and $\hat{\mathbf{x}}_{i}$. Also, $\lambda\left(\mathbf{x}_{i}, \hat{\mathbf{x}}_{i}\right)$ is the smallest eigenvalue of the matrix, $\mathbf{A}=\mathbf{B R}$, for a given pair of $\mathbf{x}_{i}$ and $\hat{\mathbf{x}}_{i}$ Here, $\mathbf{B}=\operatorname{diag}\left(b_{1}, \ldots, b_{N_{i}}\right)$ is a diagonal matrix with its $l$-th diagonal element, $l=1, \ldots, N_{i}$, defined as $b_{l}=\left|x_{k}-\hat{x}_{k}\right|^{2}$, where, $x_{k}$ and $\hat{x}_{k}$ are the $k$-th element of $\mathbf{x}_{i}$ and $\hat{\mathbf{x}}_{i}$, respectively, for which it holds that $x_{k} \neq \hat{x}_{k}$. In addition, the $N_{i} \times N_{i}$ matrix $\mathbf{R}$ is defined as, $\mathbf{R}=\sqrt{\rho_{c}} \mathbf{1}_{N_{i}, N_{i}}+\left(1-\sqrt{\rho_{c}}\right) \mathbf{I}_{N_{i}, N_{i}}$, where, $\rho_{c}$ is the Pearson product-moment correlation coefficient between any pair of the RVs $Y_{j}=d_{j}^{2}$. Note that the structure of $d_{j}$ is given in (2). Also, $\mathbf{1}_{N_{i}, N_{i}}$ denotes a $N_{i} \times N_{i}$ matrix with all of its elements equal to one. Finally, $o(\cdot)$ is the little- $o$ notation. Note that (5) is valid since the effects of small and large scale fading are statistically independent [14].

The observation of (4) shows that the computation of the $\mathrm{SER}_{i}^{+\infty}$ of the $i$-th user requires the evaluation of the expectation of (5) over all possible realization of the RV $X_{i}$. Hence, by considering that the PDF of $X_{i}$ given in (1) and neglecting the non dominant term of $o\left(\gamma^{-N_{i} L}\right)$, it holds that:

$$
\begin{aligned}
P_{i}^{+\infty}\left(\mathbf{x}_{i} \rightarrow \hat{\mathbf{x}}_{i}, \gamma\right) & =\mathrm{E}_{X_{i}}\left[P_{i}^{+\infty}\left(\mathbf{x}_{i} \rightarrow \hat{\mathbf{x}}_{i}, \gamma \mid X_{i}\right)\right] \\
& \lesssim \int_{-\infty}^{+\infty}\left(\vartheta_{i} x \gamma\right)^{-N_{i} L} f_{X_{i}}(x) \mathrm{d} x \\
& =\frac{\left(\vartheta_{i} \gamma\right)^{-N_{i} L}}{\Gamma\left(k_{i}\right) \Omega_{i}} \int_{0}^{+\infty} x^{k_{i}-N_{i} L-1} e^{-\frac{x}{\Omega_{i}}} \mathrm{~d} x
\end{aligned}
$$

where, $\Gamma(\cdot)$ is the incomplete gamma function defined in [19, p. 899]. By using the following integration formula [19, p.346, 3.381, 4], $\int_{0}^{+\infty} x^{\nu-1} e^{-\mu x} d x=\mu^{-\nu} \Gamma(\nu), \nu>0, \operatorname{Re}\{\mu\}>0$, and the fact that, $\vartheta_{i}=\lambda\left(\mathbf{x}_{i}, \hat{\mathbf{x}}_{i}\right) \sqrt[N_{i} L]{2} / 4 r_{i}^{a}$, we have that:

$$
\begin{aligned}
P_{i}^{+\infty}\left(\mathbf{x}_{i} \rightarrow \hat{\mathbf{x}}_{i}, \gamma\right) & \lesssim \frac{\Gamma\left(k_{i}-N_{i} L\right)}{\Gamma\left(k_{i}\right)} \\
& \times\left[\frac{\lambda\left(\mathbf{x}_{i}, \hat{\mathbf{x}}_{i}\right) \Omega_{i} \sqrt[N_{i} L]{2}}{4 r_{i}^{a}} \gamma\right]^{-N_{i} L},
\end{aligned}
$$

for, $k_{i}-N_{i} L>0$. Due to the fact that, $0<N_{i} \leq N_{r}$, (7) can be incorporated in (4) only when $k_{i}-N_{r} L>0$.

Since (4) is a scaled version of the summation of addends 
of the form of (7), when the value of SNR approaches infinity $(\gamma \rightarrow+\infty)$, only the addends with the smallest exponent of $\gamma$, which is $N_{i} L$, need to be considered. The smallest exponent of $\gamma$ takes place when $N_{i}=1$. In this way, (4) is further approximated as:

$$
\mathrm{SER}_{i}^{+\infty} \lesssim \frac{1}{\left|\mathcal{B}_{i}\right|} \sum_{\mathbf{x}_{i}} \sum_{\hat{\mathbf{x}}_{i} \in \mathcal{A}\left(\mathbf{x}_{i}\right)} P_{i}^{+\infty}\left(\mathbf{x}_{i} \rightarrow \hat{\mathbf{x}}_{i}, \gamma\right)
$$

where, $\mathcal{A}\left(\mathbf{x}_{i}\right)$ is the set of symbols $\hat{\mathbf{x}}_{i}$ for which it holds that: i) $\hat{\mathbf{x}}_{i} \neq \mathbf{x}_{i}$ and ii) there is only one different element between $\mathbf{x}_{i}$ and $\hat{\mathbf{x}}_{i}\left(N_{i}=1\right)$.

Since in (8) the summations are undertaken by using only the PEPs, $P_{i}^{+\infty}\left(\mathbf{x}_{i} \rightarrow \hat{\mathbf{x}}_{i}, \gamma\right)$, for which it holds that $N_{i}=$ $1,(8)$ and the analysis provided below are valid as long as $k_{i}-L>0$. This happens because, in this case, the PEP of (6) is expressed as in (7). Note that, based on the measured data and the analysis of [13], the previous condition is expected to be satisfied by a practical system implementation.

Based on the previous simplifications and with the aid of some algebraic manipulations, (8) can be further approximated as:

$$
\begin{aligned}
\mathrm{SER}_{i}^{+\infty} & \lesssim \frac{\sum_{\mathbf{x}_{i}} \sum_{\hat{\mathbf{x}}_{i} \in \mathcal{A}\left(\mathbf{x}_{i}\right)} \frac{\Gamma\left(k_{i}-L\right)}{\Gamma\left(k_{i}\right)}\left[\frac{\lambda\left(\mathbf{x}_{i}, \hat{\mathbf{x}}_{i}\right) \Omega_{i} \sqrt[L]{2}}{4 r_{i}^{a}} \gamma\right]^{-L}}{\left|\mathcal{B}_{i}\right|} \\
& =\left[\sqrt[L]{\frac{2\left|\mathcal{B}_{i}\right| \Gamma\left(k_{i}\right)}{\Gamma\left(k_{i}-L\right) \delta_{i}}} \frac{\Omega_{i}}{4 r_{i}^{a}} \gamma\right]^{-L}
\end{aligned}
$$

where, $\delta_{i}=\sum_{\mathbf{x}_{i}} \sum_{\hat{\mathbf{x}}_{i} \in \mathcal{A}\left(\mathbf{x}_{i}\right)}\left[\lambda\left(\mathbf{x}_{i}, \hat{\mathbf{x}}_{i}\right)\right]^{-L}$. From (9) and based on the definition of the diversity order and coding gain of [17], it can be concluded that the diversity order and coding gain of the $i$-th user is equal to $D_{i}=L$ and $C_{i}=\sqrt[L]{\frac{2\left|\mathcal{B}_{i}\right| \Gamma\left(k_{i}\right)}{\Gamma\left(k_{i}-L\right) \delta_{i}}} \frac{\Omega_{i}}{4 r_{i}^{a}}$, respectively.

The system diversity order and coding gain is obtained, similarly, by using (9) and expressing the high SNR approximation of the system SER as, $\mathrm{SER}_{\text {system }}^{+\infty}=\frac{1}{N_{u}} \sum_{i=1}^{N_{u}} \mathrm{SER}_{i}^{+\infty}$. Thus, by using (9), it can be shown that:

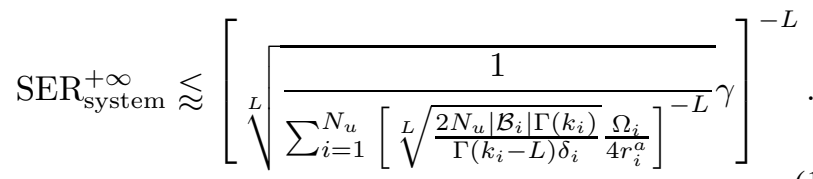

Hence, the system diversity order is equal to $D_{\text {system }}=$ $L$ and the system coding gain is equal to $C_{\text {system }}=$

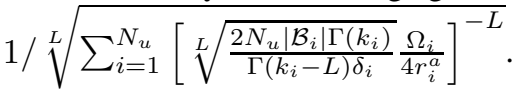

Furthermore, the observation of (9) and (10) shows that, for $k_{i}>L$, the diversity order is not affected by shadowing, both for the users' and system's performance. In contrast, the coding gain of each user and the system coding gain are affected by shadowing through the values of $k_{i}$ and $\Omega_{i}$.

As shown in Section II, SMX can be interpreted as a special case of RSM. Therefore, the analysis presented here is directly applicable to SMX by simply setting $N_{s}=N_{r}$. Since the diversity order of the $i$-th user does not depend on the value of $N_{s}$, the comparison of RSM and SMX in the high SNR
TABLE I

PERFORMANCE DIFFERENCE BETWEEN RSM AND SMX IN HIGH SNR AND BASED ON THE RATIO $\Delta_{i}, i=1$.

\begin{tabular}{c|c|c|c|c}
\hline \hline System Configuration & $N_{s}$ & $k_{\text {user }}(\mathrm{bpcu})$ & $\Delta_{1}(\mathrm{~dB})$ & $\Delta_{1}^{\text {Sim }}(\mathrm{dB})$ \\
\hline \hline$N_{t}=10, N_{r}=2, N_{u}=4$ & 1 & 4 & -1.11 & -1.3 \\
$N_{t}=20, N_{r}=4, N_{u}=4$ & 1 & 8 & -6.49 & -6.1 \\
$N_{t}=20, N_{r}=4, N_{u}=4$ & 2 & 8 & -1.35 & -1.25 \\
$N_{t}=20, N_{r}=4, N_{u}=4$ & 3 & 8 & 1.49 & 1.25 \\
$N_{t}=20, N_{r}=5, N_{u}=4$ & 4 & 10 & 1.93 & 1.25 \\
\hline \hline
\end{tabular}

can be directly obtained by evaluating the following ratio:

$$
\Delta_{i}=\frac{C_{i}^{\mathrm{RSM}}}{C_{i}^{\mathrm{SMX}}}=\sqrt[L]{\frac{\sum_{\mathbf{x}_{i} \in \mathcal{B}_{i}^{\mathrm{SMX}}} \sum_{\hat{\mathbf{x}}_{i} \in \mathcal{A}\left(\mathbf{x}_{i}\right)}\left[\lambda\left(\mathbf{x}_{i}, \hat{\mathbf{x}}_{i}\right)\right]^{-L}}{\sum_{\mathbf{x}_{i} \in \mathcal{B}_{i}^{\mathrm{RSM}}} \sum_{\hat{\mathbf{x}}_{i} \in \mathcal{A}\left(\mathbf{x}_{i}\right)}\left[\lambda\left(\mathbf{x}_{i}, \hat{\mathbf{x}}_{i}\right)\right]^{-L}} .}
$$

Here, $C_{i}^{\mathrm{RSM}}$ and $C_{i}^{\mathrm{RSM}}$ are the coding gains of the $i$-th user when RSM and SMX are deployed, respectively. Also, $\mathcal{B}_{i}^{\text {RSM }}$ and $\mathcal{B}_{i}^{\mathrm{SMX}}$ are the considered transmission alphabets of RSM and SMX, respectively. For $\Delta_{i}>1$ and in high SNR, RSM outperforms SMX, otherwise SMX performs better. In fact, in high SNR, the quantity $\Delta_{i}$ in $\mathrm{dB}$ is the performance difference between RSM and SMX. Note that, from (11), it can be concluded that the performance difference between the two transmission methods is only determined by the deployed transmission alphabets, and shadowing has no affect. The same approach can be followed to compare the system performance.

\section{RESUlts AND Discussion}

In this section, the theoretical framework developed in this paper is validated by using Monte Carlo simulation results. For all studied scenarios, the considered simulation setups are given in the captions of the corresponding figures.

In Fig. 1, the theoretical results of (9) and (10) are validated by being compared against the corresponding simulation results. The computation of the high SNR approximation of the SER of the $i$-th user is conducted by using (9). Also, the high SNR approximation of the system SER is conducted by using (10). As shown in Fig. 1, the derived diversity order follows perfectly the slope of the simulated SER curves. Furthermore, the derived coding gain is shown to be a relatively good approximation (upper bound) of the actual coding gain. The difference between the coding gain obtained via the analysis of Section III and the actual coding gain is due to the: i) use of the union bound method in (4); ii) approximated form of (5); and iii) additional approximations during the derivation between (4), (9) and (10).

Figure 2 presents the performance comparison between RSM and SMX (benchmark system) in the broadcast channel. As shown in the left sub-figure of Fig. 2, SMX performs better than RSM due to higher coding gain. However, in the right sub-figure of Fig. 2, for practical values of BER less than $10^{-2}$, RSM outperforms SMX by about $1 \mathrm{~dB}$ due to higher coding gain. This highlights that the selection of the most efficient transmission technique depends on a specific system setup. The most efficient scheme can be determined by means of simulation results or by comparing the achievable coding gains (i.e. evaluating $\Delta_{i}$ ). In order to demonstrate the accuracy of $\Delta_{i}$ in (11), the Table I presents the coding gain difference between RSM and SMX in high SNR for the user 1 using the 

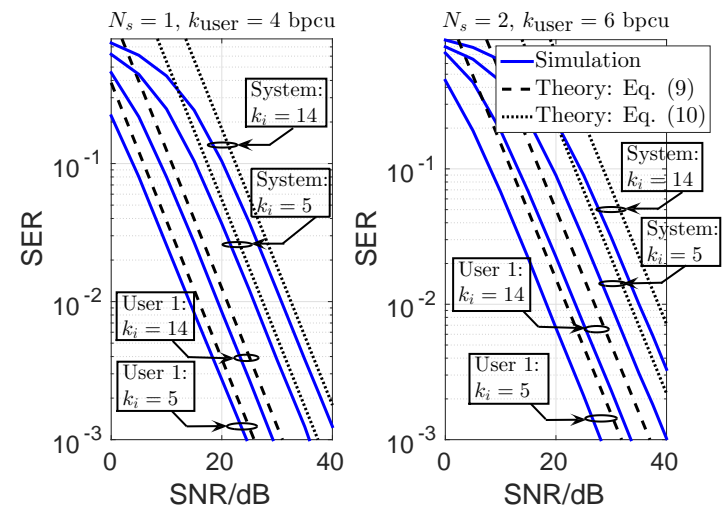

(a) Setup: $N_{t}=16, N_{r}=4, N_{u}=4, N_{s}=\{1,2\}, k_{\text {user }}=$ $\{4,6\}$ bpcu; $\Omega_{i}=2, k_{i}=5, \sigma_{X_{i}}^{2}=20$ and $\Omega_{i}=2, k_{i}=14$, $\sigma_{X_{i}}^{2}=56$, for $i=1, \ldots, 4 ; r_{1} \stackrel{X_{i}}{=} 1, r_{2}=1.5, r_{3}=1.75$, and $r_{4}=2 ;$ and $\alpha=5$.
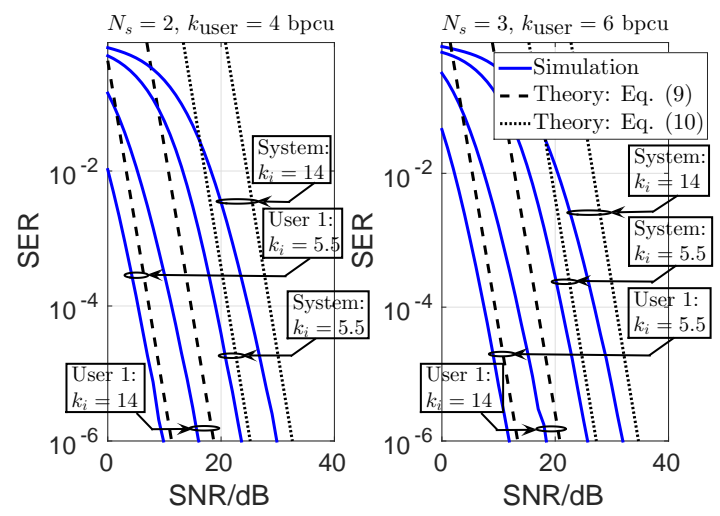

(b) Setup: $N_{t}=20, N_{r}=4, N_{u}=4, N_{s}=\{2,3\}, k_{\text {user }}=$ $\{6,8\}$ bpcu; $\Omega_{i}=2, k_{i}=5.5, \sigma_{X_{i}}^{2}=22$ and $\Omega_{i}=2, k_{i}=14$, $\sigma_{X_{i}}^{2}=56$, for $i=1, \ldots, 4 ; r_{1}=1, r_{2}=1.5, r_{3}=1.75$, and $r_{4} \stackrel{i}{=} 2 ;$ and $\alpha=5$.

Fig. 1. High SNR performance analysis of RSM: simulation results vs. the bounds in Section III.
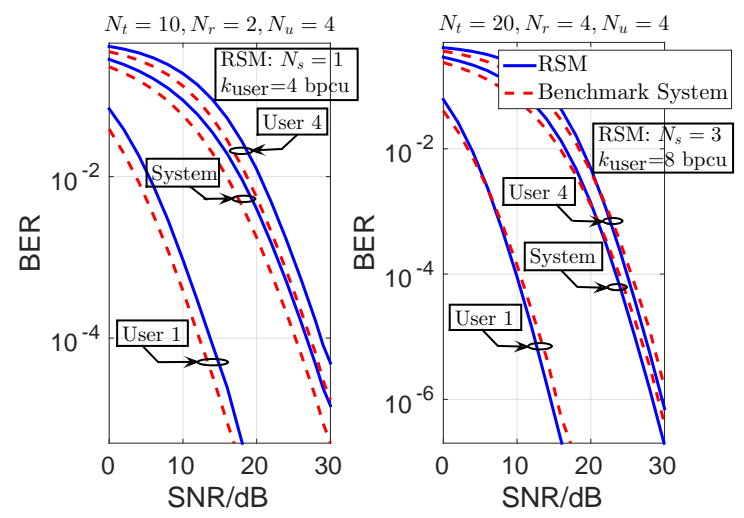

Fig. 2. Performance comparison between RSM and SMX in the MIMO broadcast channel. Setup: $N_{t}=10, N_{r}=2, N_{u}=4, k_{\text {user }}=4$ bpcu and $N_{t}=20, N_{r}=4, N_{u}=4, k_{\text {user }}=8$ bpcu $; \Omega_{i}=2, k_{i}=7, \sigma_{X_{i}}^{2}=28$, for $i=1, \ldots, 4 ; r_{1}=1, r_{2}=1.5, r_{3}=1.75$, and $r_{4}=2$; and $\alpha=5$.

framework of (11) and the actual difference $\Delta_{1}^{\text {Sim }}$ as obtained via numerical results. Note that the channel parameters for the scenarios of Table I are the same as the ones given in Fig. 2. From Table I, it can be concluded that $\Delta_{i}$ (in $\mathrm{dB}$ ) provides a close approximation of the performance difference between the two schemes.

\section{CONCLUSIONS}

In this paper, the performance of RSM in the shadowing MIMO broadcast channel was characterized in terms of its diversity order and coding gain. A theoretical criterion that determines the superiority between RSM and SMX in the shadowing MIMO broadcast channel was established. It was shown that RSM performs better than SMX in some scenarios.

\section{REFERENCES}

[1] R. Mesleh, H. Haas, S. Sinanović, C. W. Ahn, and S. Yun, "Spatial modulation," IEEE Trans. on Veh. Tech., vol. 57, no. 4, pp. 2228 2241, Jul. 2008.

[2] M. Di Renzo, H. Haas, A. Ghrayeb, S. Sugiura, and L. Hanzo, "Spatial modulation for generalized MIMO: Challenges, opportunities, and implementation," Proc. IEEE, vol. 102, no. 1, pp. 56-103, Jan 2014.

[3] A. Stavridis, S. Sinanović, M. D. Renzo., and H. Haas, "Energy evaluation of spatial modulation at a multi-antenna base station," in Proc. of the 78th IEEE Veh. Tech. Conf. (VTC), Las Vegas, USA, Sep. 2-5, 2013.

[4] J. Jeganathan, A. Ghrayeb, L. Szczecinski, and A. Ceron, "Space shift keying modulation for MIMO channels," IEEE Trans. on Wireless Commun., vol. 8, no. 7, pp. 3692-3703, Jul. 2009.

[5] M. Wen, X. Cheng, H. Poor, and B. Jiao, "Use of SSK modulation in two-way amplify-and-forward relaying," IEEE Trans. on Veh. Techn., vol. 63, no. 3, pp. 1498-1504, March 2014.

[6] P. Som and A. Chockalingam, "Performance analysis of space-shift keying in decode-and-forward multihop MIMO networks," IEEE Trans. on Veh. Techn., vol. 64, no. 1, pp. 132-146, Jan 2015.

[7] P. Yang, Y. Xiao, B. Zhang, S. Li, M. El-Hajjar, and L. Hanzo, "Power allocation-aided spatial modulation for limited-feedback MIMO systems," IEEE Trans. on Veh. Technol., vol. 64, no. 5, pp. 2198-2204, May 2015.

[8] M. Maleki, H. Bahrami, S. Beygi, M. Kafashan, and N. Tran, "Space modulation with csi: Constellation design and performance evaluation," IEEE Trans. on Vehicular Techn., vol. 62, no. 4, pp. 1623-1634, May 2013.

[9] A. Stavridis, D. Basnayaka, S. Sinanovic, M. Di Renzo, and H. Haas, "A virtual MIMO dual-hop architecture based on hybrid spatial modulation," IEEE Trans. on Commun., vol. 62, no. 9, pp. 3161-3179, Sept 2014.

[10] R. Zhang, L. L. Yang, and L. Hanzo, "Error probability and capacity analysis of generalised pre-coding aided spatial modulation," IEEE Trans. on Wireless Commun., vol. 14, no. 1, pp. 364-375, Jan 2015.

[11] C. Masouros and L. Hanzo, "Dual layered MIMO transmission for increased bandwidth efficiency," IEEE Trans. on Veh. Technol., vol. PP, no. 99, pp. 1-1, 2015.

[12] A. Stavridis, M. D. Renzo, and H. Haas, "Performance analysis of multistream receive spatial modulation in the MIMO broadcast channel," IEEE Trans. on Wireless Commun., vol. 15, no. 3, pp. 1808-1820, March 2016.

[13] A. Abdi and M. Kaveh, "On the utility of gamma PDF in modeling shadow fading (slow fading)," in Proc. of the 49th IEEE Veh. Tech. Conf. (VTC), 1999.

[14] M. Matthaiou, N. Chatzidiamantis, G. Karagiannidis, and J. Nossek, "ZF detectors over correlated K fading MIMO channels," IEEE Trans. on Commun,., vol. 59, no. 6, pp. 1591-1603, June 2011.

[15] F. Yilmaz and M. S. Alouini, "A new simple model for composite fading channels: Second order statistics and channel capacity," in Proc. of the 7th Int. Symp. on Wireless Commun. Syst., Sept 2010.

[16] F. Yilmaz and M.-S. Alouini, "A unified MGF-based capacity analysis of diversity combiners over generalized fading channels," IEEE Trans. on Commun., vol. 60, no. 3, pp. 862-875, March 2012.

[17] Z. Wang and G. Giannakis, "A simple and general parameterization quantifying performance in fading channels," IEEE Trans. on Commun., vol. 51, no. 8, pp. 1389-1398, Aug 2003.

[18] J. G. Proakis, Digital Communications, 4th ed. New York, NY, USA: McGraw-Hill, 2000.

[19] I. S. Gradshteyn and I. M. Ryzhik, Table of Integrals, Series, and Products, 7th ed., A. Jeffrey and D. Zwillinger, Eds. Academic Press, Mar. 2007, ISBN-10: 0123736374. 